\title{
Reincarnation can save Schrödinger's cat
}

It's one of the most perplexing questions in physics: how does the seemingly exotic behaviour of tiny particles in the quantum realm collapse to create the classical reality observable in matter that is at least a molecule big? Now, an experiment further muddies the distinction between the two realms by demonstrating that it is possible to halt the transition from the quantum to the classical in its tracks - and reverse it. The achievement could provide quantum computing with a crucial capability (See 'A fix for quantum computers').

According to the 'Copenhagen interpretation' of quantum mechanics, arrived at in 1927 , observing a quantum object disturbs its state, causing it to flit from quantum to classical realities. Before they are measured, atoms and subatomic particles do not have fixed properties, they exist in a 'superposition' in which they have many mutually contradictory properties.

The notion is exemplified by the paradox of Schrödinger's cat, a thought experiment in which a cat is locked in a box with a vial of poisonous gas that would be broken if a quantum particle was in one state, and remain intact if the particle was in another. While the box is closed, the particle exists in a superposition of both states simultaneously, so the poison must also simultaneously be both released and contained, and, in turn, the cat must be both alive and dead. When the box is opened, the quantum superposition collapses, and the cat is either killed or saved, in an instant.

Now, Nadav Katz at the University of California, Santa Barbara, and his colleagues have performed an experiment in which they pull a quantum state back from the brink of collapse, 'uncollapsing' it and returning it to its unobserved state. Effectively, they have peeked at Schrodinger's cat in its box, but saved it from near-certain death (N. Katz et al. http://arxiv. org/abs/0806.3547).

To physicists raised on the textbook Copenhagen interpretation, any notion of uncollapsing a quantum state seems "astonishing", says Markus Büttiker, a quantum physicist at the University of Geneva in Switzerland. "On opening the box, Schrödinger's cat is either dead or alive - there is no in between."

However, a more recent interpretation of quantum mechanics, 'decoherence theory', suggests that collapse does not occur instantaneously. Instead it plays out gradually as the quantum system slowly interacts with its environment (see Nature 453, 22-25; 2008). In 2006, Alexander Korotkov of the University of California, Riverside, and Andrew Jordan, of the University of Rochester in New York, proposed that this may leave open a time period in which experimenters could intervene to halt the collapse (A. N. Korotkov \& A. N. Jordan Phys. Rev. Lett. 97, 166805; 2006). They provided blueprints for an experiment to test the idea, which Katz, Korotkov and their colleagues have now done.

\section{Living dead}

In place of Schrödinger's cat, Katz and his colleagues created a 'phase qubit', often used in quantum computing experiments, involving a superconducting circuit that is broken by an insulating junction. The qubit is made up of fluctuations in the average current flowing around this loop and is characterized by its 'phase', the degree that the current gets knocked out of step as it crosses the junction.

The qubit can have two different energies, high and low, and the team prepared the qubit so that it took on a superposition of both energy values simultaneously - an experimental equivalent of Schrödinger's cat being simultaneously alive and dead. Any attempt to measure the qubit's energy directly would permanently collapse the state into one of these two energy values - effectively opening

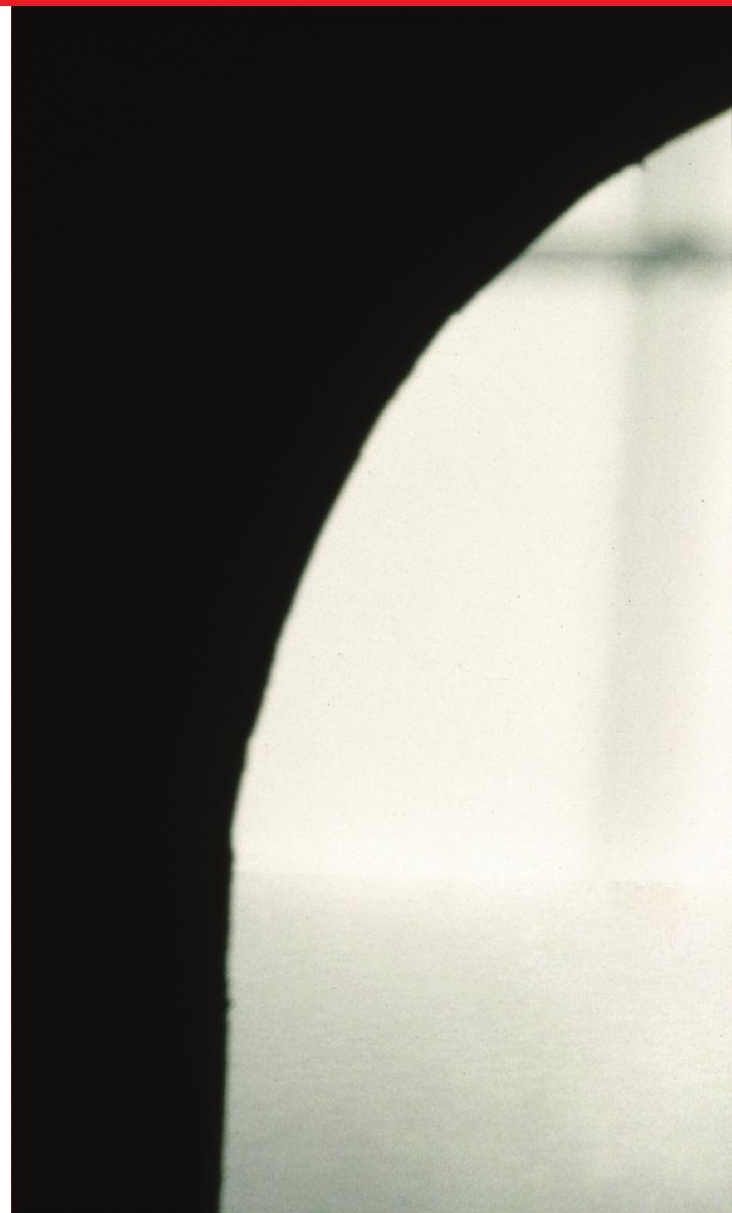

Schrödinger's box. The trick is to avoid this total collapse by sneakily getting an indirect hint of the qubit's energy, explains Katz.

The team turned to a quantum property $\Sigma$ known as 'tunnelling', whereby quantum particles can overcome energy barriers when they seem to have too little energy to do so.

In this case, the team changed the average current running through the circuit to control the 'height' of a 'barrier' that would make it harder for the qubit to change phase. The barrier was strong enough to prevent a low-energy qubit from changing to a new phase, but a highenergy qubit state could be tempted to make

\section{A fix for quantum computers}

Quantum computers promise of both states simultaneously. to outperform their classical counterparts - but they are tough to construct. However, the ability to 'uncollapse' quantum states demonstrated by Nadav Katz at the University of California, Santa Barbara, and his colleagues could now give those attempting to build such computers a boost.

Conventional computers process information in 'bits' that take on values of 0 or 1. Quantum computers, by contrast, use 'qubits' that can also exist in 'superpositions'
This means that a quantum computer can carry out multiple calculations in parallel. But so far, only a relatively small number of qubits have been combined to perform calculations in the lab.

One of the biggest problems plaguing those hoping to build large-scale quantum computers is that qubits are extremely fragile. Even a tiny interference from the outside world can knock a qubit out of whack, collapsing its quantum state and leading to loss of stored information. The demonstration that it is possible to rescue a collapsing qubit, 'uncollapse' it and return it to its original quantum state could one day be used to rectify errors before information is lost.

Robin Blume-Kohout, a quantum theorist at the Perimeter Institute for Theoretical Physics in Ontario, Canada, agrees. "This might be a very useful error-correction technique for quantum information processing down the road."

Z.M. 


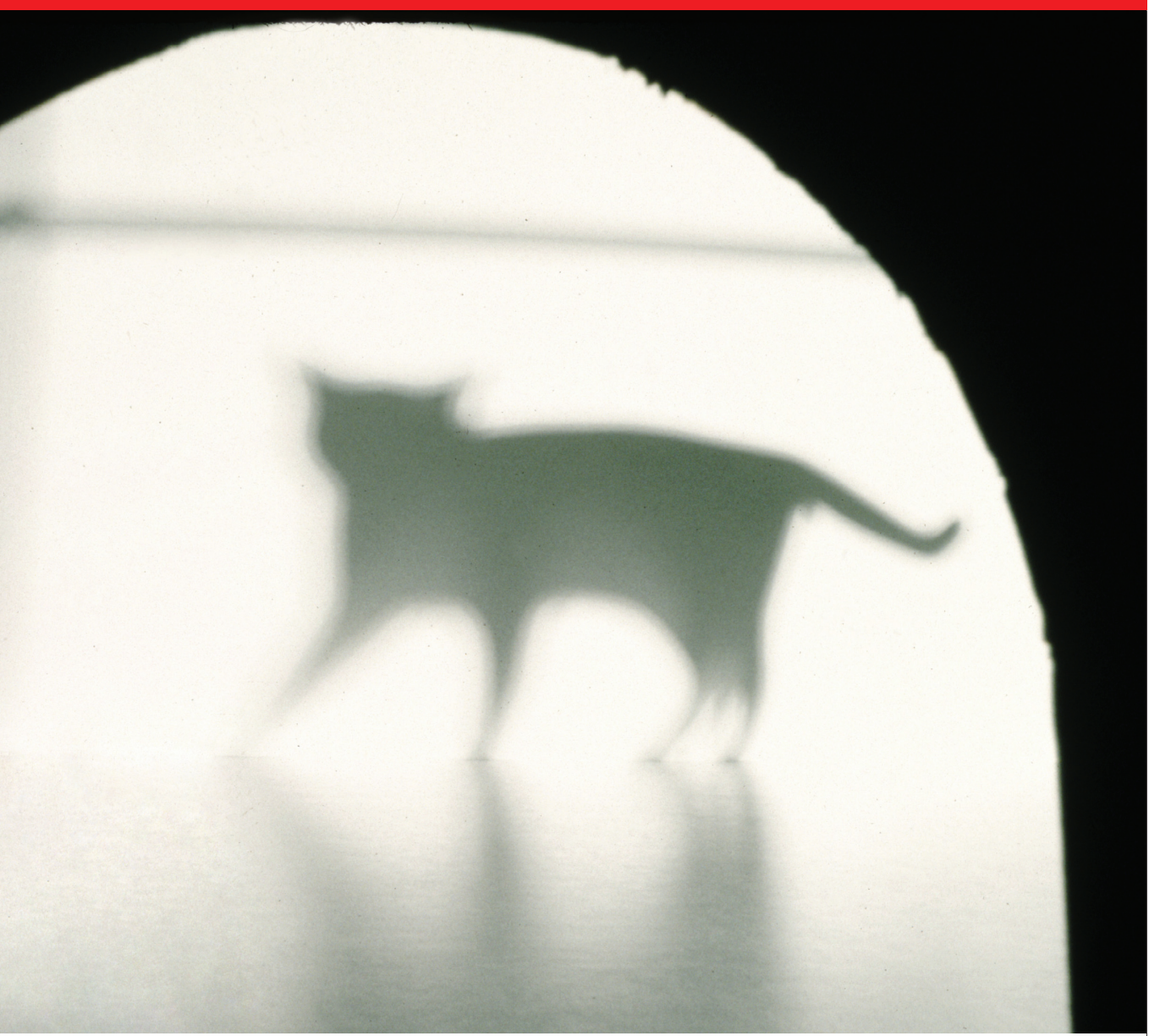

the transition. So, looking at whether the qubit made the transition - creating a telltale burst of magnetic energy - can reveal something about the energy state of the qubit.

The least exciting result is if the qubit succeeds, says Katz. This means that it definitely fully collapsed into its high-energy state and tunnelled. “That's game over," says Katz. It's the equivalent of fully opening the box, seeing that the cat is definitely alive, and releasing it.

\section{Time travel}

It's when the qubit does not tunnel that things become more interesting. This means there is a good chance that it was in the lower level. However, crucially, you cannot know for sure, so this measurement does not completely collapse the system, says Katz. "We have peeked at the cat and then quickly shut the lid."

This 'weak' measurement does slightly disturb the system, however. If the experiment ended there, this disturbance would be enough to slowly drive the qubit towards a total collapse into the lower energy state, says Katz. His team has statistically confirmed this outcome by repeating the measurement on thousands of similarly prepared qubits and then terminating the experiment at this point. Measuring the qubit's state at this stage reveals that in the vast majority of cases, it collapses to the lower energy state. That is, on reopening the box, the cat is most likely to be found dead.

To uncollapse the state, the team had to catch the qubit before it completed this journey to complete collapse and "undo the damage that we had done", says Katz. They used a standard technique to swap the qubit's energy levels, by firing a specially prepared microwave pulse at it - turning a qubit in a high-energy level to one in a low level, and vice versa. Because of the swap, when they then repeated their weak measurement, they caused a disturbance that exactly cancelled the effects of their first measurement. The first time they peeked, they nudged the cat towards death, but the second time they nudged it towards life, leaving it back where it started, explains Katz.

Once again, the team confirmed statistically that the state had been returned to its original uncollapsed form - with a roughly equal probability of collapsing into either energy level by performing multiple runs of the experiment. Measuring the state at the end of the experiment, they found that it was just as likely to be found in either the high- or low-energy state.

"The data are clear," says Büttiker. "This is a breakthrough experiment."

The result is a warning that our understanding of how classical reality emerges may be naive, says Vlatko Vedral, a quantum physicist at the University of Leeds, UK. "It tells us that we really can't assume that measurements create reality, because it is possible to erase the effects of a measurement and start again."

"The quantum world has become more tangible, and the nature of reality even more mysterious," adds quantum theorist Maximilian Schlosshauer of the University of Melbourne in Australia.

Zeeya Merali 